\title{
Evaluación educativa utilizando la metodología Ranking Task en la enseñanza de la mecánica estelar en la asignatura de Introducción a la Astronomía Optativa
}

Joel Alemán Ramírez

\section{Resumen}

El presente trabajo muestra los resultados obtenidos en cuanto a ganancia en conocimientos por estudiantes del espacio pedagógico denominado Introducción a la Astronomía después de haber incorporado el Ranking Task como herramienta de aprendizaje colaborativa.

Este estudio se realizó en ciudad universitaria a finales del año académico 2012 e inicios del año 2013, tomando como sujetos de estudio un grupo de 26 estudiantes con las características típicas de estudiantes que cursan esta asignatura general y optativa, el estudio se declara exploratorio su interés es conocer el comportamiento de los estudiantes ante este nuevo recurso de enseñanza aprendizaje.

El estudio se considera aún parcial debido a las interioridades propias del proceso de investigación, el espacio pedagógico y los sujetos de estudio por lo que se espera una nueva oportunidad para validar los hallazgos del presente estudio.

Palabras clave: Aprendizaje colaborativo, constructivismo, tareas de posicionamiento

\section{Abstract}

This paper shows results obtained in terms of gain in knowledge by students of pedagogical space called Introduction to Astronomy after adding Ranking Task as collaborative learning tool.

This study was conducted on campus at the end of the academic year 2012 and early 2013, taking as subjects a group of 20 students with the typical characteristics of 
students taking this course elective general, and exploratory study declares interest is to understand the behavior of students at this new teaching and learning resource.

The study is still considered part because the internals own research process, educational space and study subjects so expect a new opportunity to validate the findings of this study.

Keywords: Collaborative learning, constructivism, ranking task

Joel Alemán Ramírez (joel.aleman@UNAH.edu.hn) Departamento de Astronomía y Astrofísica, Facultad de Ciencias Espaciales, Universidad Nacional Autónoma de Honduras. 


\section{Introducción}

El nuevo modelo educativo de la UNAH, demanda nuevas estrategias en los procesos de enseñanza aprendizaje exige, estrategias de corte constructivista y centradas en el estudiante que posibiliten el cambio cognitivo y el desarrollo de competencias que preparen a los futuros egresados a este "nuevo mundo" de constante cambio. Se hace necesario entonces escoger aquellos recursos de aprendizaje que se ajusten mejor a las exigencias impuestas en el modelo educativo, es por ello que en este estudio se comienza por estudiar si el Ranking Task (RT) llena estos requisitos.

Este estudio trata sobre las experiencias obtenidas en la utilización de esta herramienta en la enseñanza de la astronomía. Se hace en primer lugar una revisión conceptual de la herramienta, haciendo referencia a los estudios más significativos que fundamentan sus aplicaciones en la enseñanza de la ciencia en general y de la astronomía en particular, seguidamente se presentan las bases psicopedagógicas que fundamentan el RT como recurso constructivista de enseñanza aprendizaje, luego se pasa revisión a los instrumentos que servirán para recoger la información en el estudio, continua el documento con la metodología de investigación pasando inmediatamente a los resultados, su discusión y algunas conclusiones que sirvan para justificar (o no) la incorporación del RT en el espacio pedagógico de Introducción a la Astronomía.

\section{¿Por qué incorporar Ranking Task en el espacio pedagógico de Intro- ducción a la Astronomía?}

El Ranking Task (RT) del inglés: Ranking, ordenamiento y Task, tarea es decir; tareas de posicionamiento, son herramientas pedagógicas utilizadas para valorar aspectos conceptuales del aprendizaje.

A través de baterías de ejercicios sobre un mismo tema se presentan una serie de situaciones físicas que varían muy poco entre sí y que deben ser resueltas de manera conjunta por grupos de estudiantes. Se atribuye a Maloney (Maloney, 1987) como el primero en dar una descripción de esta herramienta. Éste autor, menciona que el RT se fundamenta en Reglas de Evaluación, una técnica muy útil que por lo regular se utilizan en pruebas diagnósticas (del tipo pre y post test) sobre todo en actividades de laboratorio y/o tareas.

Estudios posteriores revelan la utilidad del RT en estrategias de enseñanza colaborativa en el aula (Maloney \& Friedel, 1996) a este respecto Maloney, en el 
mismo estudio de 1987 encontró que estudiantes trabajando en pequeños grupos con RT desarrollan una estrategia de resolución de problemas caracterizada por tres elementos claramente diferenciados:

\section{Descripción del problema $\rightarrow$ construcción de una base conceptual $\rightarrow$ Cálculo}

Llegando a este último a través de un proceso pasó a paso hasta encontrar la solución al problema.

Si bien la herramienta de RT esta categorizada como de corte constructivista, su aplicación no está generalizada de hecho, su estudio y aplicación en el área de enseñanza de la astronomía es muy incipiente aún y la mayoría de los estudios hechos al respecto son extranjeros sin tenerse conocimiento de algún estudio de este tipo en Honduras.

Originalmente la herramienta ha sido utilizada para la enseñanza de la física de donde parte la mayoría de las aplicaciones y estudios sobre su utilidad en los diferentes espacios pedagógicos asociados.

En Ciudad Universitaria probablemente su introducción como herramienta de enseñanza sea debido al PhD. Armando Euceda quien la introdujera como parte del contenido del curso de Enseñanza de la Física en el año 2009, asignatura obligatoria del Postgrado en Física General de la UNAH.

Su aplicación como herramienta de evaluación de aprendizajes no forma parte aun del portafolio de recursos de enseñanza aprendizaje de profesores de la UNAH y su aplicación se limita a experiencias aisladas de profesores de la escuela de física en forma de tareas para la casa; y por un profesor de astronomía que regularmente la introduce como actividad de aprendizaje colaborativa en la segunda unidad del pensum del curso de general y optativo de Introducción a la Astronomía. En los anexos de este documento se adjunta una batería de instrumentos RT para un tema en particular de los abordados en el estudio.

\section{Bases psicopedagógicas del RT}

Se mencionaba párrafos arriba el RT como herramienta de enseñanza aprendizaje constructivista, enfoque de aprendizaje que se establece como norma en el nuevo modelo educativo de la UNAH.

En estos nuevos enfoques de aprendizaje, el constructivista hace hincapié en la importancia que la interacción entre personas y las situaciones tiene en la ad- 
quisición de conocimientos y el desarrollo de competencias (Cobb \& Bowers, 1999) es decir, el supuesto fundamental de constructivismo es considerar al estudiante como elemento activo en el proceso de enseñanza aprendizaje.

El constructivismo sin embargo, ha tenido implicaciones no solo en la apreciación de la adquisición de competencias y conocimientos sino también en los medios y métodos utilizados para tal fin es decir, en aspectos del desarrollo curricular y la instrucción en si misma (currículo integrado) base hoy en día de las estrategias centradas en el estudiante.

En el caso particular de la enseñanza de la astronomía diferentes estudios revelan la importancia del enfoque constructivista. Estudios como los de BonweIl y Eison (Bonwell \& Eison, 1991), Prather (Prather, Slater, \& Adams, 2004) entre otros, exponen la mejora en la comprensión de contenidos de los estudiantes cuando estos hacen uso de estrategias de enseñanza participativa centrados en el estudiante.

Resulta más fácil sin embargo decirlo que hacerlo y es que la mayoría de profesores que hayan tenido encuentros con estas herramientas de aprendizaje constructivistas saben del esfuerzo y tiempo que se requiere el desarrollar materiales apropiados así como de su implementación en el aula.

Esto no es algo nuevo o propio de profesores de física yl astronomía de la UNAH, es algo que ya ha sido señalado en numerosos estudios. En el área de enseñanza de la astronomía estos estudios como los de (Brissenden, Slater, \& Mathieu, 2002) han demostrado que son tres las grandes prioridades al respecto:

1. Elaboración de materiales curriculares basados en la investigación

2. La evaluación cuantitativa de la eficacia de estos materiales en el aula

\section{Estrategias de enseñanza}

Elementos que aun hoy en día no han sido suficientemente atendidos. Otra teoría cognitiva que además del constructivismo fundamente el desarrollo de materiales curriculares como el RT es la Teoría de Esquemas.

Estas teorías tienen que ver en como organizan la información que reciben los estudiante alrededor de un esquema pre elaborado (Stein \& Trabasso, 1982) 0 a través de ideas conectadas entre sí (Slavin, 1988). 
En la teoría del esquema se estudia la compleja interacción entre la imaginería visual y las estructuras de conocimiento que se crean y organizan activamente: normas, proposiciones, listas de palabras, conceptos de categorización y conocimiento procedimental que tienen lugar en la mente de cada persona.

Una definición de esquema puede encontrase en Schunk (Schunk, 2012) citando a Anderson (Anderson, 1990) que expone esta teoría como una estructura que organiza grandes cantidades de información en un sistema significativo. En el mismo texto (Schunk, 2012) se menciona otra definición que va muy acorde con las RT: El esquema es un estereotipo que determina una pauta general o secuencia de pasos asociada con un concepto, destreza o acontecimiento particular.

\section{Descripción de los instrumentos RT}

Para el presente estudio se han utilizado instrumentos RT, diseñados para obtener inicialmente concepciones alternativas que comúnmente utilizan los estudiantes para explicar fenómenos físicos (y astronómicos) estos instrumentos representan una serie de ejercicios conceptuales que proporcionan un primer camino para que los estudiantes puedan construir nuevos conocimientos. Los instrumentos han sido adaptados de otros que se encuentran disponibles en internet los cuales son utilizados comúnmente para propósitos educativos. Estos, se basan en la teoría de esquemas y cubren los temas en que los estudiantes muestran mayor dificultad de comprensión.

Los instrumentos abordan un mismo tema desde diferentes enfoques en diferentes niveles de complejidad elevándose poco a poco de un instrumento a otro. El diseño de estos instrumentos y su utilización en investigación educativa relacionada con la enseñanza de la ciencia están validados por diferentes estudios hechos al respecto por ejemplo (Shulman, 1986), (Grayson, 2004), que evalúan las dificultades específicas y las concepciones alternativas y las estrategias a que recurren los estudiantes para hacer frente a la problemática presentada.

Se trabajaron ocho temas para los cuales se adaptaron los instrumentos, estos se aplicaron en un pilotaje con estudiantes de una sección de astronomía y el acompañamiento de docentes bilingües del departamento el objetivo fue evaluar la traducción, la redacción, la claridad de la tarea solicitada entre otros. Después de esto se hicieron las correcciones respectivas y se aplicaron en el estudio. 
Se identificaron ocho temas:

1. Movimiento de las estrellas en el cielo

2. Fases de la luna

3. Las estaciones

4. Leyes de Kepler

5. Principio de gravitación universal

6. Luminosidad de las Estrellas

\section{Efecto Doppler}

8. Magnitud y Distancia.

Para cada tema se dispusieron cinco RT donde se resolvieron tareas de diferentes grados de dificultad. Las tareas más elementales consistieron en encontrar soluciones cualitativas a los problemas presentados exigiendo de los estudiantes reflexiones más profundas, confrontando así, la tarea memorística a la que suelen hacer uso como estrategia de aprendizaje procurando con ello el cambio conceptual esperado. Posteriores tareas requirieron soluciones cuantitativas cuyo cálculo se basó en valores dispuestos en tablas o diagramas.

La variación en la presentación de la tarea problema, obliga a los estudiantes a apropiarse del concepto a través de diferentes vías, facilitando con ello el desarrollo de la comprensión de una manera más integral. En algunos casos además de las tareas de carácter cualitativo y de cálculo, se ha solicitado al estudiante la exposición de los motivos que lo obligaron a tomar las decisiones en la resolución del problema con ello, se asegura la apropiación de la ley física en juego y como esta puede ser aplicada en la predicción del resultado.

\section{Metodología}

El grupo de estudio lo conformaban 26 estudiantes del curso general y optativo de Introducción a la Astronomía. Estos constituyen una muestra representativa de la población de estudiantes universitarios que cursaron la asignatura en el periodo que se realizó el estudio compuesta por alumnos de tres secciones 
activas con una población total de 80 estudiantes(es decir el 33\% de la población estudiantil).

Esta población se caracteriza por:

- Diversidad de género

- Diversidad de edad

- Diversidad de estudios previos

- $\quad$ Diversidad de intereses profesionales

La selección de esta muestra se basó en el formato de muestreo no probabilístico intencionado, siendo los criterios y juicios de valor considerados los siguientes:

1. El profesor tiene que tener conocimientos y disposición de uso de la aplicación de RT y conocimientos especializados de astronomía.

2. Conocimiento y experiencia del docente en conducción de investigaciones bajo el formato de investigación-acción.

Se estructuró una prueba diagnóstica con 28 preguntas de selección múltiple las que cubrían los temas tratados en el estudio, dicha prueba se aplicó a todos los estudiantes el primer día de clases. Estas mismas preguntas se utilizaron en las evaluación posteriores a la instrucción tradicional, también sirvieron para formar las evaluaciones de salida (con sutiles variaciones) después de haber introducido las RT en el aula.

La instrucción tradicional se entiende para propósitos de este estudio constituida por clase magistral acompañada de demostraciones apoyada en multimedios además de lecturas en casa y experiencias situadas en el observatorio astronómico.

Después de haber tomado la evaluación de salida se incorporan las RT y se forman grupos de tres y cuatro estudiantes, grupos formados por afinidad y que permanecieron juntos hasta el final del estudio. 
La actividad con las RT llevó en promedio 20 minutos al final de los cuales se aplicó la evaluación de salida respectiva (evaluación post Ranking Task).

En los anexos de este documento se adjuntan un ejemplo de los instrumentos (RT) utilizados.

\section{Resultados}

Se midió la comprensión del estudiante en los temas abordados en el presente estudio antes y después de la instrucción tradicional y luego de incorporar la herramienta RT.

De acuerdo a la experiencia tenida en cursos anteriores de introducción de astronomía, se esperaba que la ganancia obtenida con la instrucción tradicional no fuese muy grande. Se observó que la puntuación fue en promedio del $23 \%$ de aciertos antes de la instrucción, llegando a una media del 29\% de respuestas correctas después de la instrucción tradicional, en cada uno de los temas abordados en el estudio. En la tabla siguiente se muestran estos resultados así como también los obtenidos después de RT

\begin{tabular}{|l|l|c|c|c|}
\hline \multirow{2}{*}{ No. } & \multirow{2}{*}{ Tema } & \multicolumn{3}{|c|}{ Promedios en los aciertos } \\
\cline { 3 - 5 } & $\mid$ & $\begin{array}{c}\text { Evaluación Diag- } \\
\text { nóstica }\end{array}$ & $\begin{array}{c}\text { Instrucción Tra- } \\
\text { dicional }\end{array}$ & Instrucción con RT \\
\hline 1 & Movimiento del Cielo & 19 & 35 & 42 \\
\hline 2 & Fases de la Luna & 19 & 23 & 35 \\
\hline 3 & Estaciones & 42 & 46 & 50 \\
\hline 4 & Leyes de Kepler & 19 & 23 & 38 \\
\hline 5 & Gravitación & 19 & 27 & 31 \\
\hline 6 & Magnitud y Distancia & 12 & 19 & 23 \\
\hline 7 & Luminosidad de Estrellas & 35 & 38 & 50 \\
\hline 8 & Efecto Doppler & 15 & 23 & 31 \\
\hline & Promedio & 23 & 29 & 38 \\
\hline
\end{tabular}

Tabla 1: resultados promedios de aciertos en evaluaciones de entrada - salida en ocho temas principales del curso de introducción a la astronomía 
El comportamiento experimentado en cada uno de los temas puede notarse mucho mejor haciendo uso del siguiente gráfico:

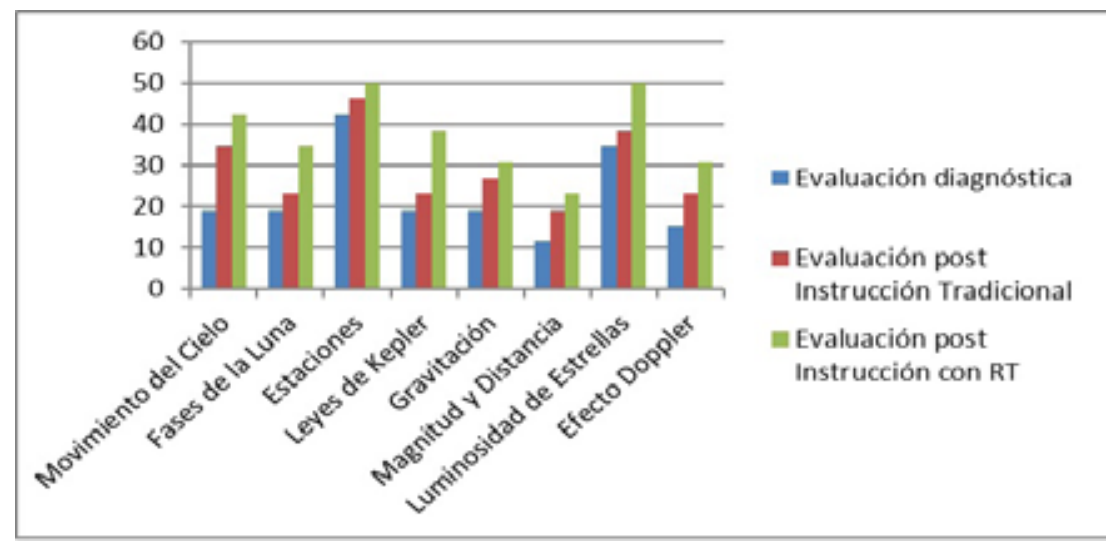

Gráfico 1: resultados promedios de aciertos en las evaluaciones de entrada-salida en ocho temas Principales de Introducción a la Astronomía optativa.

Los resultados anteriores muestran el aspecto cuantitativo del estudio, otra parte que se tomó en cuenta fue el grado de aceptación de los estudiantes a trabajar con la herramienta, el $82 \%$ de los estudiantes consultados consideran el RT como una herramienta importante para su aprendizaje ya que les permite aclarar puntos relacionados con la teoría que no les quedaron suficientemente claros con la exposición del profesor. Otro aspecto que valoraron mucho fue la estrategia de trabajo en equipo ya que cerca del $90 \%$ de ellos considera que esta estrategia de enseñanza en más "dinámica" y les permite relacionarse con sus otros compañeros y le ayuda a mantenerse atentos a las explicaciones del profesor. Aun así, hubo aspectos valorados negativamente siendo el más importante el tiempo que lleva el realizar una experiencia con RT en el aula ya que según los estudiantes (cerca del $80 \%$ ) la hora clase es muy corta para realizar la experiencia y por último, un pequeño porcentaje $(9 \%)$ preferiría realizar otro tipo de actividad en el aula.

\section{Discusión}

Los resultados que se muestran aquí son muy conservadores aún si se comparan, con los resultados obtenidos en investigaciones sobre las ganancias de conocimientos hechos en estudiantes de Introducción a la Astronomía después de la instrucción tradicional (Prather, Slater, \& Adams, 2004). Si bien dichos estudios 
se han realizado en el extranjero, el análisis minucioso de dichos trabajos revela semejanzas en cuanto a la temática abordada, el estudiante objeto de estudio y las características de enseñanza aprendizaje tradicional abordadas en el curso de Introducción a la Astronomía de la UNAH.

Ahora bien, el objetivo general de este estudio es explorar el comportamiento de los estudiantes ante una nueva estrategia de enseñanza aprendizaje como lo es el Ranking Task, aspectos como la ganancia en conocimientos, la interacción entre pares, la elección del momento propicio, entre otros son elemento importantes a considerar si se está pensando en incorporar este recurso. Se ha evaluado los dos primeros elementos obteniéndose los resultados que previamente se han mostrado. Habría que esperar que la introducción del RT redundara en una mejora en la ganancia de conocimientos que resulto cierto (38\%)

Puede observarse de la tabla 1 y gráfico 1 anteriores que después de la incorporación del RT como recurso de enseñanza aprendizaje tuvo lugar un aumento en el porcentaje de aciertos logrados por los estudiantes en cada uno de los ocho temas que forman parte del estudio. Si bien los resultados son conservadores, quince puntos si se considera la relación entre evaluación diagnostica y la evaluación después de incorporar RT y de seis entre la evaluación diagnostica y la evaluación post instrucción tradicional, tómese en cuenta que el trabajo desarrollado colaborativamente entre los estudiantes en una cuantas horas (trabajando con RT) fue significativamente más importante que la instrucción tradicional de hecho, la diferencia en la ganancia en conocimientos entre una y otra fue muy similar, llegando a ser incluso mayor para RT (9 puntos) como se muestra en la tabla. Para comprender mejor lo que se quiere decir con esto, piénsese que la ganancia obtenida a través de la instrucción tradicional ha sido posible después de toda una serie de estrategias y recursos preparados para tal fin (clases magistrales, recursos multimedia lectura en casa, etc) en comparación con el trabajo colaborativo de los estudiantes.

Los resultados aquí encontrados si bien muestran que después de la aplicación de RT hay una mejora en la comprensión de los temas estudiados, debe tenerse en cuenta entre otras cosas, el estudiante objeto de estudio así como la naturaleza intrínseca del curso de Introducción a la Astronomía Optativa para llegar a tener análisis más completo. En este sentido, la aplicación de esta herramienta en un curso de especialidad y con estudiantes de carrera podría arrojar resultados diferentes que los obtenidos con estudiantes, de primero y segundo año, de diversas carreras e intereses, de un curso optativo general como lo es el espacio pedagógico de Introducción a la Astronomía optativa. Elementos que representan 
una limitante en cuanto al desarrollo y comprensión de contenidos por parte de estudiantes que se matriculan en el curso.

\section{Conclusiones}

La incorporación de una herramienta como Ranking Task, redunda en una ganancia en conocimientos de los estudiantes y es una estrategia de aprendizaje colaborativa que es bien recibida por los estudiantes. Al ser una herramienta de desarrollo y evaluación de conceptos puede incorporarse en cualquier fase del proceso de enseñanza aprendizaje deberá, estudiarse el momento propicio y la planificación adecuada en cuanto al número de ítems y duración de la actividad.

Al no ser Introducción a la Astronomía optativa, un curso de formación específica (especializada, de formación profesional) y debido a que muchos de los estudiantes matriculados no tienen formación en el área fisicomatématica, se debe incorporar estrategias de enseñanza aprendizaje que contribuyan a la formación general y al desarrollo de competencias en el análisis de documentos científicos más que desarrollar competencias en el manejo de operadores matemáticos para el modelado de fenómenos astronómicos; la herramienta RT se convierte así una buena alternativa para ser incorporada en el portafolio de recursos del profesor de Introducción a la Astronomía optativa.

\section{Agradecimientos}

El autor del presente artículo agradece al departamento de astronomía y astrofísica por su apoyo en el desarrollo del estudio.

A los docentes del departamento de astronomía y astrofísica que participaron en la validación de los instrumentos para la investigación.

A los estudiantes de Introducción a la Astronomía Optativa, objeto del presente estudio.

\section{Bibliografía}

- Anderson, J. R. (1990). Cognitive Psycology and its implications. 3th ed. N. Y: Freeman. 
- Bonwell, C., \& Eison, J. (1991). Active learning: Creating excitement in the classroom. Washington, DC: ASHE.: ASHE-ERIC Higher Education Report No. 1

- Brissenden, G., Slater, T. F., \& Mathieu, R. D. (2002). The Role of Assessment in the Development of the College Introductory Astronomy Course. Astronomy Education Review , 1(1), 1.

- Cobb, P., \& Bowers, J. (1999). Cognitive and Situate learning perspectives in theory and practice. Educational Researcher, 28(2), 4 - 15.

- Grayson, D. (2004). Concept substitution: A teaching strategy for helping students disentangle related physics concepts. American Journal of Physics, , 72(8), 1126-1133.

- Maloney, D. P. ( 1987). Ranking tasks: A new type of test item. . Journal of College, 16(6), 510.

- Maloney, D. P., \& Friedel, A. W. (1996). Ranking tasks revisited. Journal of College. Journal of College, 25, 205-210.

- Prather, E. E., Slater, T. F., \& Adams, J. (2004). The use and effectiveness of lecture:Tutorials in introductory astronomy courses. Astronomy Education Review.

- $\quad$ Prather, E., Slater, T., \& Adams, J. (2004). The use and effectiveness of lecture:Tutorials in introductory astronomy courses. Astronomy Education Review., 3(1), 122.

- Schunk, D. H. (2012). Teorías del Aprendizaje: una perspectiva educativa. Sexta edición. México D.F., México: Pearson.

- Shulman, L. (1986). Those who understand: Knowledge growth in teaching. Education Research, 15(2), 4-14.

- Slavin, R. E. (1988). Educational psychology: Theory into practice. . Englewood Cliffs, NJ, USA: Prentice Hall. 
- Stein, N., \& Trabasso, T. (1982). What's in a story? An approach to comprehension and instruction. Advances in instructional psychology, (pp. 213-267) (Vol.2).

\section{Glosario}

\section{Aprendizaje Colaborativo}

El aprendizaje colaborativo es "...un sistema de interacciones cuidadosamente diseñado que organiza e induce la influencia recíproca entre los integrantes de un equipo."(Johnson y Johnson, 1998). Se desarrolla a través de un proceso gradual en el que cada miembro y todos se sienten mutuamente comprometidos con el aprendizaje de los demás generando una interdependencia positiva que no implique competencia.

El Aprendizaje Colaborativo se adquiere a través del empleo de métodos de trabajo grupal caracterizado por la interacción y el aporte de todos en la construcción del conocimiento.

\section{Constructivismo}

- Los individuos aprenden mejor cuando construyen activamente el conocimiento y la comprensión.

- Enfatizan los contextos sociales del aprendizaje y afirman que el conocimiento es tanto edificado como construido.

\section{Muestreo No Probabilístico}

En este tipo de muestras, también llamadas muestras dirigidas o intencionales, la elección de los elementos no depende de la probabilidad sino de las condiciones que permiten hacer el muestreo (acceso o disponibilidad, conveniencia, etc).

Es más conveniente usar un muestreo no probabilístico, por ejemplo cuando vamos a hacer estudios de casos, de poblaciones heterogéneas, o en estudios que son dirigidos a poblaciones y grupos muy específicos donde la interesa una cuidadosa y controlada selección de sujetos con determinadas características. 